\title{
The TAK1-JNK cascade is required for IRF3 function in the innate immune response
}

Bianhong Zhang ${ }^{1,}$, Meng Li ${ }^{1, *}$, Liang Chen ${ }^{1}$, Kai Yang ${ }^{1}$, Yufei Shan ${ }^{1}$, Lianhui Zhu ${ }^{1}$, Shaogang Sun ${ }^{1}$ Lin $\mathrm{Li}^{1}$, Chen Wang ${ }^{1}$

${ }^{I}$ Laboratory of Molecular Cell Biology, Institute of Biochemistry and Cell Biology, Shanghai Institutes for Biological Sciences, Chinese Academy of Sciences, 320 Yue Yang Road, Shanghai 200031, China

Interferon regulatory factor (IRF) 3 is critical for the transcriptional induction of chemokines and cytokines during viral or bacterial invasion. The kinases Tank binding kinase (TBK)1 and Ikappa B kinase (IKK)\& can phosphorylate the C-terminal part of IRF3 and play important roles in IRF3 activation. In this study, we show that another kinase, c-Jun- $\mathrm{NH}_{2}$-terminal kinase (JNK), phosphorylates IRF3 on its N-terminal serine 173 residue, and TAK1 can stimulate IRF3 phosphorylation via JNK. JNK specific inhibitor SP600125 inhibits the N-terminal phosphorylation without affecting the C-terminal phosphorylation. In addition, IRF3-mediated gene expressions on lipopolysaccharide (LPS) or polyinosinic-cytidylic acid (polyI:C) treatment are severely impaired by SP600125, as well as for reporter gene assay of IRF3 activation. Knockdown of TAK1 further confirmed these observations. Interestingly, constitutive active IRF3(5D) can be inhibited by SP600125; JNK1 can synergize the action of IRF3(5D), but not the S173AIRF3(5D) mutant. More importantly, polyI:C failed to induce the phosphorylation of mutant S173A and SP600125 dramatically abrogated IRF3 phosphorylation and dimerization that was stimulated by polyI:C. Thus, this study demonstrates that the TAK1-JNK cascade is required for IRF3 function, in addition to TBK1/IKKE, uncovering a new mechanism for mitogen-activated protein (MAP) kinase to regulate the innate immunity.

Keywords: JNK, TAK1, IRF3, innate immunity

Cell Research (2009) 19:412-428. doi: 10.1038/cr.2009.8; published online 20 January 2009

\section{Introduction}

Pattern-recognition receptors (e.g., Toll-like receptors, or TLRs) detect the presence of microbial invasion and trigger a complex program of gene expressions, which ultimately provoke a series of immune responses. In humans, 10 TLRs have been identified [1,2]. For example, in the presence of CD14 and MD2, TLR4 can recognize the Gram-negative bacterial cell wall components such as lipopolysaccharide (LPS) [3], whereas TLR3 responds to the virus-derived double-stranded RNA (dsRNA). Polyinosinic-cytidylic acid (polyI:C) is a dsRNA mimetic and can also be recognized by TLR3 $[4,5]$. In addition,

*The two authors contributed equally to this work. Correspondence: Chen Wang ${ }^{\mathrm{a}}$, Lin $\mathrm{Li}^{\mathrm{b}}$

${ }^{\mathrm{a}}$ Tel: +86-21-54921185, E-mail: cwang01@sibs.ac.cn

${ }^{\mathrm{b}} \mathrm{Tel}$ : +86-21-54921188, E-mail: 1li@sibs.ac.cn

Received 25 September 2008; revised 26 September 2008; accepted 27 September 2008; published online 20 January 2009 a new paradigm for monitoring cytoplasmic microbes is emerging and has been delineated over the past several years [6]. LPS or dsRNA can activate multiple signaling pathways, including interferon regulatory factor (IRF)3, $\mathrm{NF}-\kappa \mathrm{B}$, and mitogen-activated protein kinase (MAPK) pathways, to participate in the transcriptional induction of chemokines and cytokines, which include interferons (IFNs), RANTES, and a subset of IFN-stimulated genes (ISGs). Activation of IRF3 is central to these gene expressions [7-9].

The role of IRF3 (interferon regulatory factor 3) activation in the innate immune response has been intensively explored in the recent years. Signaling pathways and several key receptors/adaptors have been characterized. IRF3 activation by LPS and polyI:C occurs in a Toll/IL$1 \mathrm{R}$ domain-containing, adaptor-inducing IFN- $\beta$ (TRIF)dependent manner $[5,10]$. On dsRNA binding, TLR3 recruits adapter protein TRIF. TLR4 can also recruit TRIF indirectly through interaction with another adaptor called TRAM (TRIF-related adaptor molecule) [11, 12]. 
Consequently, TRIF recruits TRAF3 and non-canonical $\mathrm{I}_{\kappa} \mathrm{B}$ kinases Tank binding kinase 1 (TBK1) and Ikappa B kinase (IKK) $\varepsilon$, which phosphorylate IRF3 at several serine/threonine residues of its $\mathrm{C}$-terminal trans-activation domain $[13,14]$. Although redundant in vitro, TBK1 and IKK $\varepsilon$ display functional differences in vivo. TBK1 could play a major role in IFN- $\beta$ induction. Indeed, studies with TBK1/IKKE-deficient MEF and macrophage point to a more important role for TBK1 than for IKK $\varepsilon$ in IFN induction in response to LPS, dsRNA and virus infection $[15,16]$. It remains controversial whether TRIF can recruit IKK $\varepsilon$ or not $[8,13]$.

IRF3 is a 427-amino-acid phosphoprotein that is constitutively expressed in two forms (forms I and II) and localizes in the cytoplasm in unstimulated cells. On viral infection, IRF3 is phosphorylated within the C-terminus of the protein on serine $385,386,396,398,402,405$, and threonine 404, resulting in hyper-phosphorylated forms III and IV $[17,18]$. Originally, it was widely accepted that the C-terminal phosphorylation elicited a conformational change that allowed the formation of the IRF3 dimer, which translocated to the nucleus and formed a transcriptional complex with the coactivator $\mathrm{CBP} / \mathrm{p} 300$ [17-20]. However, alternative phosphorylations at other residues have been confirmed to play an important role in the biological activation of IRF3 [21, 22]. Several recent studies also reported a lack of correlation between nuclear accumulation of IRF3 and its hyper-phosphorylation state $[23,24]$. The particular kinase(s) responsible for this regulation remains unknown.

Interestingly, more signaling pathways were implicated in the regulation of IRF3 activation. For example, IKK $\beta$ was reported to phosphorylate the C-terminus of IRF3 and activate the IFN-stimulated response element [25]. Sarkar et al. [26] observed that the PI3K-AKT pathway accounted for partial IRF3 phosphorylation and played an essential role in dsRNA signaling. However, neither PI3K nor AKT could phosphorylate IRF3 per se. Another report revealed that $\mathrm{cPKCs}$ played a role in the synthesis of IFN- $\beta$ induced via both TLR-dependent and -independent pathways [27]. In addition, apoptosis signal-regulating kinase (ASK) 1 kinase activity was also shown to be essential for IRF3-mediated ISG induction by LPS [28]. However, these investigations did not address which forms of IRF3 phosphorylation were affected by cPKCs or ASK1.

In addition to TBK1 and IKK $\beta$, Toll-like receptors also stimulated signaling cascades that led (via TIR adapters) to the activation of MAPK pathways [8, 29]. There are three main families of MAPKs in mammals: extracellular signal-regulated kinase (ERK), p38 MAPK, and c-Jun- $\mathrm{NH}_{2}$-terminal kinase (JNK) [30], all of which are known to be activated by LPS and polyI:C. They have been shown to mediate important physiological and pathological functions in innate immunity [31, 32]. However, it remains largely unknown whether the MAPK kinases have a critical role in the regulation of IRF3 activation.

This investigation revealed that JNK can phosphorylate IRF3, and that TAK1 was the upstream kinase of this regulation. Interestingly, this phosphorylation targeted the N-terminal serine 173 residue of IRF3 instead of its canonical C-terminal segment, which accounted for the generation of IRF3 form II. In contrast, ERK1, ERK2, and p38 did not display any effect on IRF3 phosphorylation. Further study demonstrated that SP600125, a specific inhibitor for JNK, could severely impair IRF3mediated gene expression induced by LPS and polyI:C. This also held true for IRF3 reporter gene assay. Knockdown of TAK1 again confirmed these observations. Interestingly, constitutive active IRF3(5D) can be inhibited by SP600125; JNK1 and polyI:C can synergize the action of IRF3(5D), but not the S173A-IRF3(5D) mutant. Importantly, SP600125 dramatically abrogated IRF3 phosphorylation and dimerization stimulated by polyI:C. However, SP600125 had no inhibitory effect on TBK1induced C-terminal phosphorylation of IRF3. Thus, this investigation demonstrates that the TAK1-JNK cascade is required for IRF3 function in addition to TBK1/IKKE, which uncovers a new mechanism for MAPK to regulate innate immunity.

\section{Results}

\section{TAK1, JNK1 and JNK2 can stimulate phosphorylation of IRF3}

A series of recent studies established that TBK1, IKK $\varepsilon$, and IKK $\beta$ could phosphorylate IRF3 at its C-terminus and activate the IFN-stimulated response element $[13,14,25]$. IRF3 was constitutively expressed in two forms (form I and II). Form I was un-phosphorylated, whereas form II represented basal IRF3 phosphorylation $[17,18]$. This was confirmed in our experiment by treating the immunoprecipitated IRF3 isolated from cells that overexpressed IRF3 with calf intestine alkaline phosphatase (Figure 1A). Virus infection resulted in hyperphosphorylated forms of IRF3 (form III and IV) that originated at the C-terminus of IRF3 [17, 18].

To explore whether MAPK played a direct role in the regulation of IRF3, we tested whether any of the MAPKs would influence the phosphorylation status of IRF3. As expected, expression of IRF3 along with IKK $\beta$ or TBK1 could significantly promote hyper-phosphorylation (forms III and IV) of IRF3 (Figure 1B, lane 5, and Figure 1C, 
A
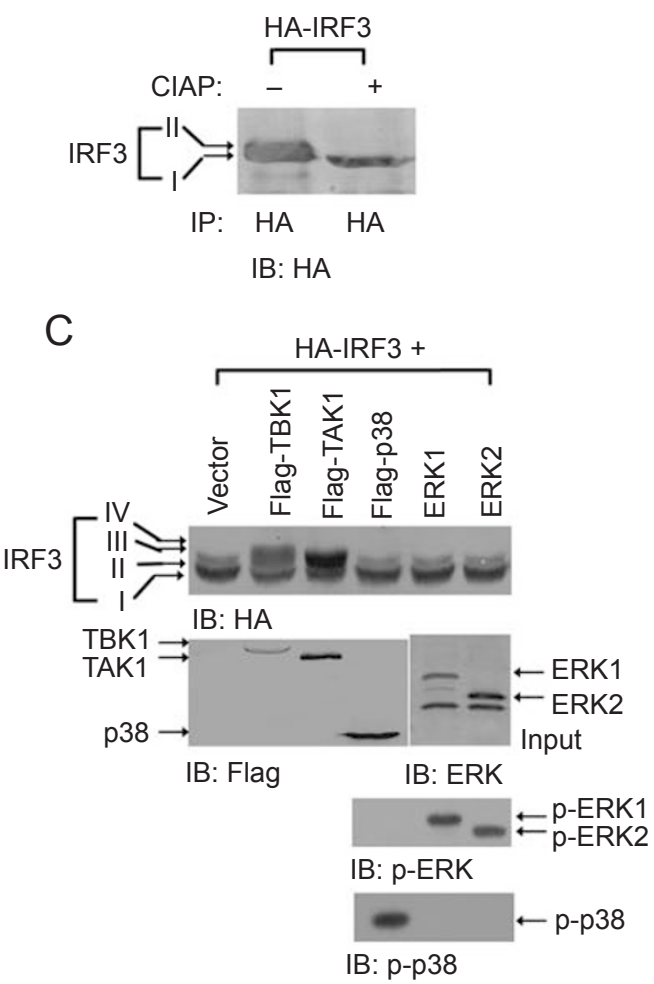

$\mathrm{E}$

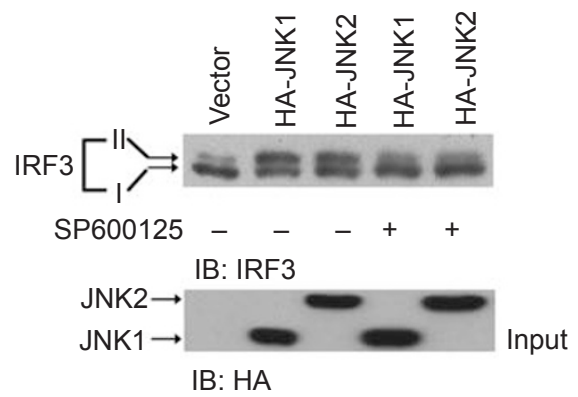

G

IB: IRF3

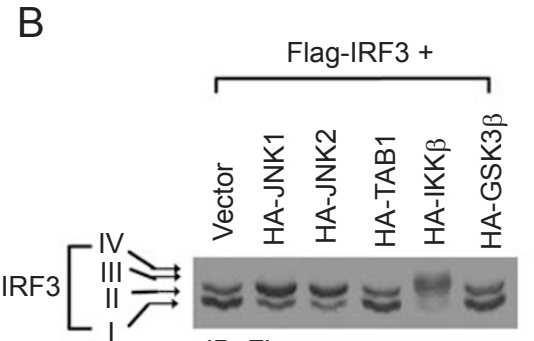

IB: Flag

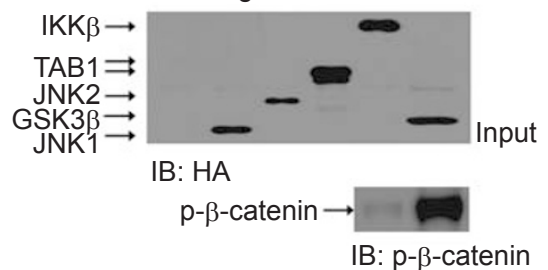

D

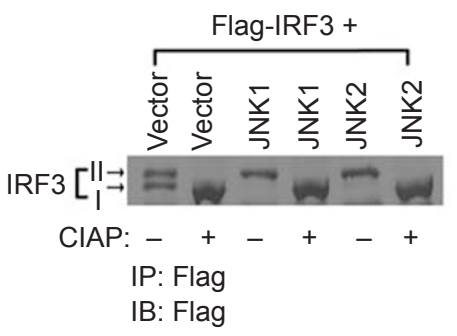

$\mathrm{F}$

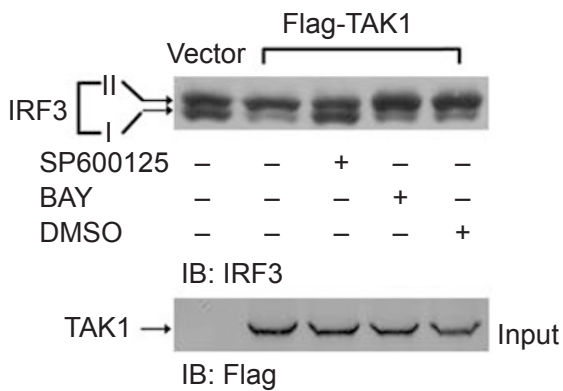

Figure 1 TAK1, JNK1, and JNK2 stimulate IRF3 phosphorylation. (A) 293T cells were transfected with HA-IRF3. 24 h after transfection, whole cell extracts were prepared and subjected to immunoprecipitation using anti-HA antibody conjugated to protein A/G beads. Immunoprecipitated IRF3 was then left untreated ( - ) or treated $(+)$ with calf intestine alkaline phosphatase and analyzed by immunoblotting using anti-HA antibody. (B) Flag-IRF3 or (C) HA-IRF3 was co-transfected into 293 T cells with the indicated constructs. $24 \mathrm{~h}$ after transfection, cells were lysed and analyzed by immunoblotting using the indicated antibodies. Middle panels, the expression of various constructs in 293T cells. Lower panels illustrate the activation of various kinases. (D) Flag-IRF3 was co-transfected into 293T cells with the indicated plasmids. $24 \mathrm{~h}$ after transfection, whole cell extracts were prepared and subjected to immunoprecipitation using anti-Flag antibody. Immunoprecipitated IRF3 was then left untreated $(-)$ or treated $(+)$ with CIAP and analyzed by immunoblotting using anti-Flag antibody. (E, F) 293T cells were transfected with the indicated constructs. $16 \mathrm{~h}$ after transfection, cells were treated with the indicated pharmacological inhibitors for $8 \mathrm{~h}$ and then lysed and probed with indicated antibodies. (G) 293T cells were treated with the indicated inhibitors for $16 \mathrm{~h}$ and then harvested and probed with anti-IRF3 antibodies. 
lane 2), which was consistent with previous reports [25]. In contrast, GSK $3 \beta$ could not alter the phosphorylation status of IRF3 (Figure 1B, lane 6), although it could phosphorylate its authentic substrate, $\beta$-catenin (Figure 1B, lower panel). Surprisingly, expression of IRF3 together with JNK1 or JNK2 was able to significantly change the ratio between forms II and I (Figure 1B, lanes 2 and 3), resulting in the predominant accumulation of the constitutive phosphorylated form of IRF3 (form II). Interestingly, JNK1 or JNK2 could not induce the formation of hyper-phosphorylated IRF3 (forms III and IV), suggesting that JNK1 and JNK2 performed a function distinct from those of TBK1 and IKK $\beta$. In addition, TAK1 behaved similarly to JNK and stimulated the generation of form II IRF3 (Figure 1C, lane 3), indicating that TAK 1 and JNK may be functionally related in terms of IRF3 regulation. However, TAB1 was not able to stimulate IRF3 phosphorylation (Figure 1B, lane 4), although it was reported to stimulate TAK1 kinase activity on cytokine treatment $[33,34]$. Like GSK3 $\beta$, other MAPKs, such as p38, ERK1, and ERK2, failed to stimulate phosphorylation of IRF3 (Figure 1C, lanes 4, 5, 6), suggesting that JNK played a unique role for IRF3. Treating the immunoprecipitated IRF3 isolated from cells co-expressing IRF3 and JNK with calf intestine alkaline phosphatase further confirmed that form II of IRF3 induced by JNK was the phosphoprotein (Figure 1D).

Alternatively, we explored endogenous IRF3 phosphorylation induced by JNK1, JNK2, or TAK1. As expected, both JNK1 and JNK2 induced a change of endogenous IRF3 from form I to form II. Furthermore, this activity was severely impaired by SP600125, a specific pharmacological inhibitor of JNK [35], indicating that JNK1 and JNK2 were responsible for the formation of form II phosphorylation (Figure 1E). Likewise, TAK1 could elicit endogenous IRF3 phosphorylation (form II), which was specifically blocked by SP600125. In contrast, neither BAY (a specific NF- $\kappa$ B inhibitor) [36] nor dimethyl sulfoxide (DMSO) could inhibit IRF3 phosphorylation (Figure 1F). These results implicated that TAK1 was the upstream kinase and phosphorylated IRF3 via JNK. Furthermore, only SP600125 markedly affected basal IRF3 phosphorylation, whereas PD98059 (ERK inhibitor), SB202190 (p38 inhibitor), BAY, or DMSO had no such effect (Figure 1G). Taken together, these data indicate that JNK1, JNK2, and TAK1 are candidate kinases responsible for the generation of form II IRF3 phosphoproteins, which was distinct from the actions of TBK1 and IKK $\beta$.

JNK can directly phosphorylate IRF3 on its N-terminal serine 173 residue
LPS could induce JNK activation via the formation of a TRAF6-TAB1-TAK1 complex [37, 38]. TAK1 is an upstream MAP3K that could phosphorylate MAP2K, which, in turn, activated the JNK kinase [38]. We wondered whether JNK was activated by TAK1 and then phosphorylated IRF3. To explore this hypothesis, we performed in vitro kinase assay to determine whether IRF3 was the authentic substrate. Therefore, these kinases were expressed in $293 \mathrm{~T}$ cells individually or in combination. The cell lysates were immunoprecipitated with specific antibodies and subjected to kinase assay using purified recombinant GST-IRF3 (wild type) as a substrate. Both JNK1 and JNK2 could directly catalyze the phosphorylation of IRF3. Co-expression of TAK1 markedly enhanced this kinase activity (Figure 2A). In contrast, TAK1 alone failed to phosphorylate IRF3 per se (Figure 2A, lane 6), which strongly suggests that IRF3 is an authentic substrate of JNK and this phosphorylation is promoted by the upstream kinase TAK1.

As reported previously, forms III and IV of IRF3 were generated by the phosphorylation of a cluster of serine residues in the $\mathrm{C}$-terminal region by TBK1 and IKK $\beta$ $[17,25]$. The above observation suggested that JNK1, JNK2, or TAK1 did not phosphorylate the same region. To substantiate this possibility, we used the IRF3(5D) mutant protein as a substrate for in vivo phosphorylation, in which the serine-threonine cluster at 396-405aa was mutated to phosphomimetic Asp(5D). We found that IRF3(5D) was no longer phosphorylated by IKK $\beta$. In contrast, phosphorylation of IRF3(5D) remained the same as that of the wild type when stimulated by JNK1, JNK2, or TAK1 (Figure 2B). The slower migration band disappeared upon treatment with calf intestine alkaline phosphatase, confirming the identity of JNK-induced phosphorylation (Figure 2C). To further map the domain of IRF3 targeted by JNK1, JNK2, and TAK1, a series of IRF3 truncation mutants were generated (Figure 2D). The in vivo phosphorylation analysis by co-expression indicated that $\mathrm{C}$-terminal truncation mutants of IRF3 (1-201 and 1-357) did not alter the generation of forms I and II. However, the IRF3 truncation mutant (1-147) lost the ability to be phosphorylated when it was coexpressed with JNK1, JNK2, or TAK1, as evidenced by the single form of IRF3. In addition, we made N-terminal truncation mutants of IRF3 (141-427, 201-427, and 240427 ) and found that JNK1, JNK2, and TAK1 could stimulate phosphorylation of the 141-427 truncation mutant. However, they failed to do so with respect to the 201-427 and 240-427 (Figure 2E). Taken together, these data solidly established that JNK1, JNK2, and TAK1 stimulated the phosphorylation of IRF3 at its N-terminus, spanning a region from aa 147 to aa 201, which was different from 


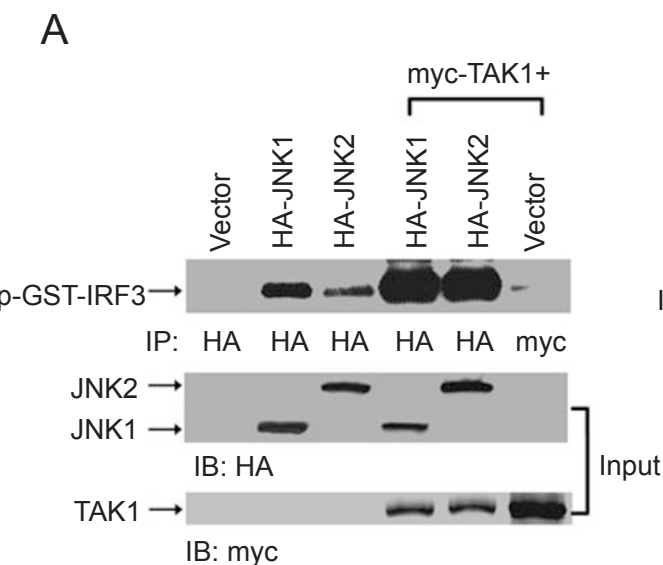

C

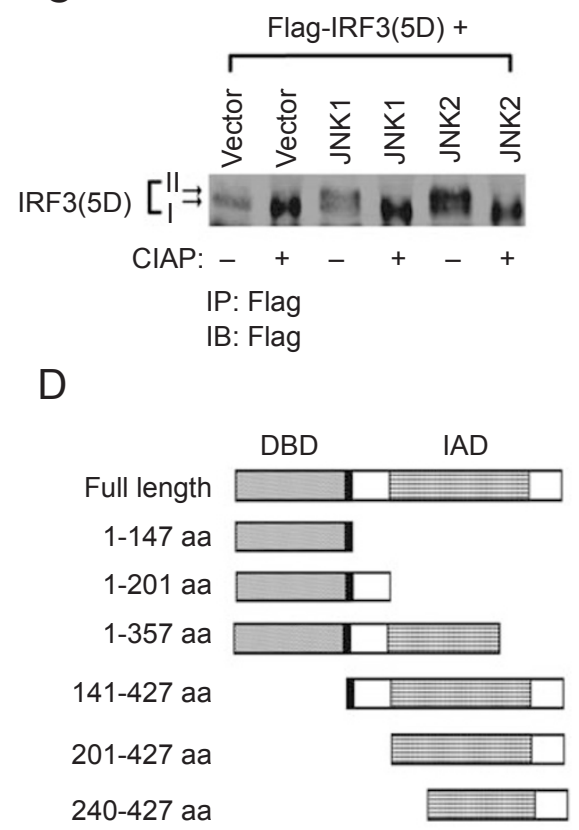

$\mathrm{B}$

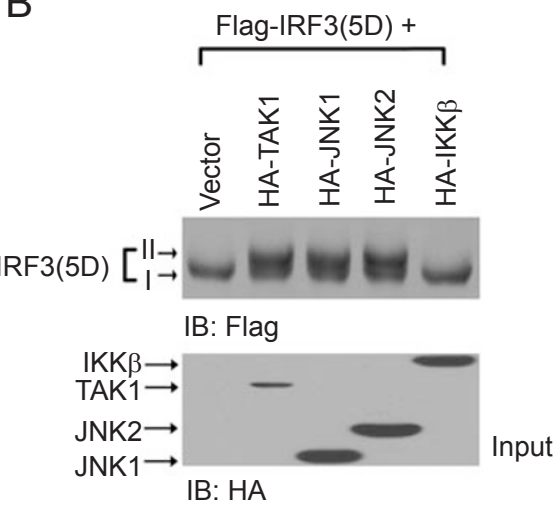

E

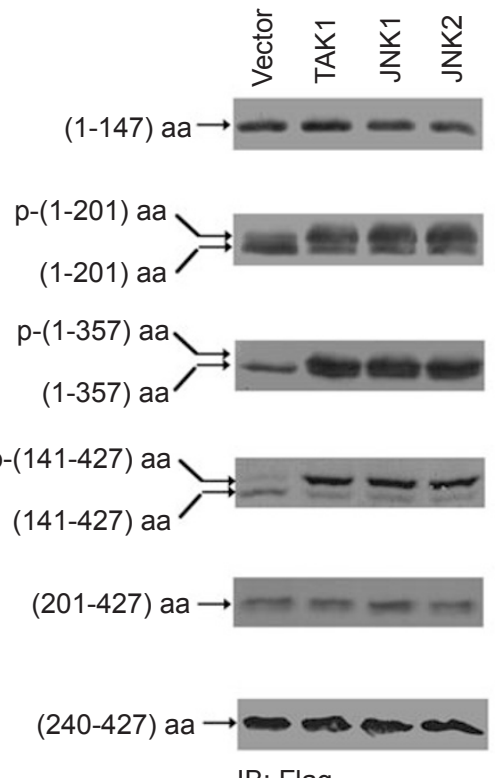

IB: Flag

Figure 2 TAK1, JNK1, and JNK2 promote IRF3 phosphorylation at its N-terminal segment. (A) 293T cells were transfected with the indicated constructs. $24 \mathrm{~h}$ after transfection, whole cell extracts were prepared and immunoprecipitated using the indicated antibodies.An kinase assay was performed as described under Materials and Methods. The expression of various constructs was confirmed using the indicated antibodies (middle and lower panels). (B) Flag-IRF3(5D) was co-transfected into $293 \mathrm{~T}$ cells with the indicated plasmids. $24 \mathrm{~h}$ after transfection, cells were lysed and analyzed by immunoblotting using the indicated antibodies. (C) Flag-IRF3(5D) was co-transfected into 293T cells with the indicated plasmids. $24 \mathrm{~h}$ after transfection, cell lysates were immunoprecipitated. The immunoprecipitated IRF3(5D) was treated with or without calf intestine alkaline phosphatase as indicated and then analyzed by immunoblotting using anti-Flag antibody. (D) Schematic diagram of IRF3 truncation mutants used in this study. (E) Mock, TAK1, JNK1, or JNK2 was transiently transfected into 293 T cells together with the indicated Flag-IRF3 truncation mutants. Then, the truncation mutants were immunoblotted with anti-Flag antibodies.

the phospho-receptor region for TBK1 and IKK $\beta$.

Only five serine/threonine residues exist in the region from aa 147 to aa 201 (S159, S173, S175, S188, and T180). We compared the protein sequence of this region between mice and humans and found that only the serine
173 residue is conserved (Figure 3A). To further identify the site(s) of phosphorylation by JNK, we constructed a series of point mutants of serine/threonine residues (S159A, S173A, S175A, S188A, and T180A). As shown in Figure 3B, JNK1 can still stimulate form II of S159A, 


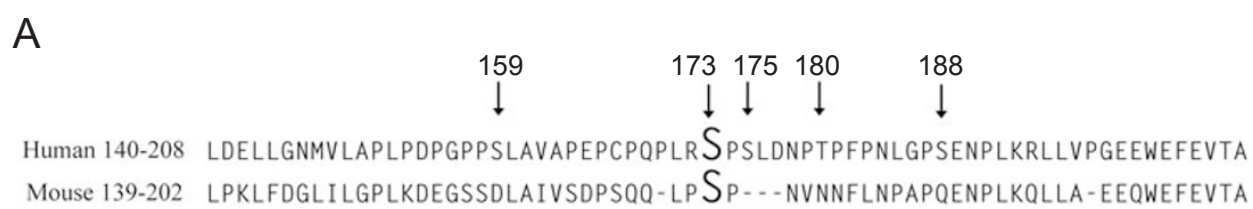

B
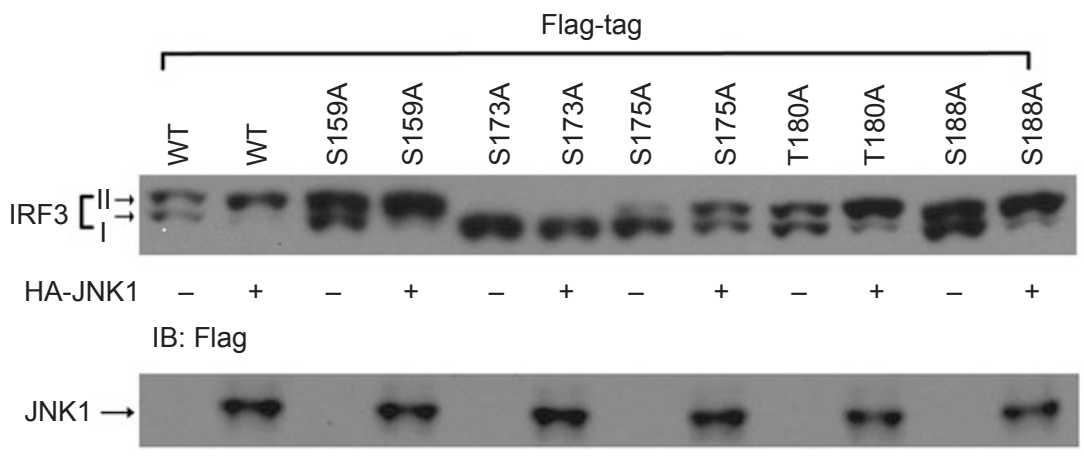

IB: HA

C

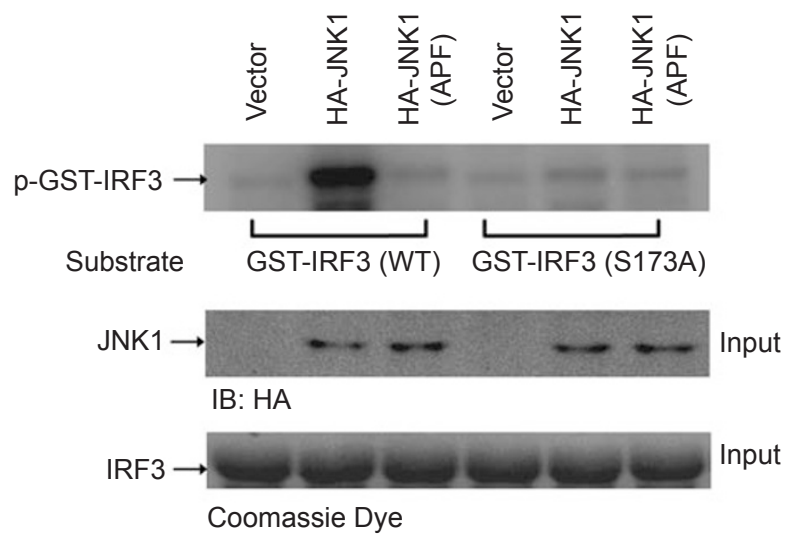

Figure 3 JNK phosphorylate on serine 173 residue of IRF3. (A) The alignment of region 140-208 between humans and mice. Only serine 173 residue is conserved among five serine/threonine residues. (B) 293T cells were co-transfected with the indicated constructs. Then the point mutants were immunoblotted with anti-Flag antibodies (upper panels) and the expression of JNK1 was monitored with anti-HA antibodies (lower panels). (C) 293T cells were transfected with the indicated constructs. Whole cell extracts were immunoprecipitated with anti-HA antibody. Then, in vitro kinase assay was performed using GSTIRF3 or GST-IRF3 (S173A) as a substrate. The expression of JNK was confirmed using anti-HA antibodies (middle panels) and the amount of the substrates was monitored by Coomassie dyes (lower panels).

S175A, S188A, and T180A mutants. Significantly, JNK1 failed to induce the generation of form II with respect to the S173A mutant (Figure 3B). Furthermore, we also performed an in vitro kinase assay to test whether the S173A mutant of IRF3 could be phosphorylated by JNK1. As expected, JNK1 promoted the phosphorylation of wildtype IRF3 (Figure 3C, lane 2). In contrast, JNK1 failed to catalyze the phosphorylation of the S173A mutant (Figure 3C, lane 5). Likewise, neither wild type nor S173A mu- tant of IRF3 could be phosphorylated by JNK1 (APF), a dominant-negative mutant of JNK1 (Figure 3C, lanes 3 and 6). Taken together, these results uncovered a new MAPK pathway that could directly phosphorylate IRF3 on its serine 173 residue.

$J N K$ regulates IRF3-mediated gene expression induced by LPS and polyI:C

Thus far, it has been established that JNK1 and JNK2 
catalyze IRF3 phosphorylation in a site distinct from those by TBK 1 and IKK $\beta$. We therefore went on to address whether this unique phosphorylation plays a physiological role in the regulation of IRF3 function. Although JNK could also stimulate the transcriptional activity of another transcription factor, alkaline phosphatase (AP-1), the PRD III-I-Luciferase reporter was responsive only to IRF3 activation in the context of LPS stimulation [39]. As shown in Figure 4A, luciferase analysis revealed that inhibition of endogenous JNK activation by SP600125 significantly impaired gene expression induced by LPS, which reduced the expression level below the basal level. However, BAY (a specific NF- $\kappa \mathrm{B}$ inhibitor) and DMSO displayed no inhibitory effect on PRD III-I-driven gene expression. In addition to IRF3, LPS could also trigger the activation of $\mathrm{NF}-\kappa \mathrm{B}$ to regulate downstream gene expression $[8,29]$. However, SP600125 had no effect on
$\mathrm{NF}-\kappa \mathrm{B}$-driven gene expression induced by LPS (Figure 4B).

As an alternative approach, we perturbed the IRF3 phosphorylation by small interfering RNA (siRNA). The endogenous expression of JNK1 or JNK2 could be knocked down individually. However, for unknown reasons, we were unable to efficiently knock down the endogenous expression of both JNK1 and JNK2 at the same time. Fortunately, an effective siRNA sequence was obtained to explore the role of TAK1 during IRF3 activation (Figure 4C). As expected, a decrease of endogenous TAK1 expression markedly diminished PRD III-I-driven gene expression induced by LPS (Figure 4D). These data indicate that the TAK1-JNK cascade positively regulates IRF3 activation in a specific way.

To further corroborate the above observation, we investigated via reverse transcription-PCR whether JNK would
A

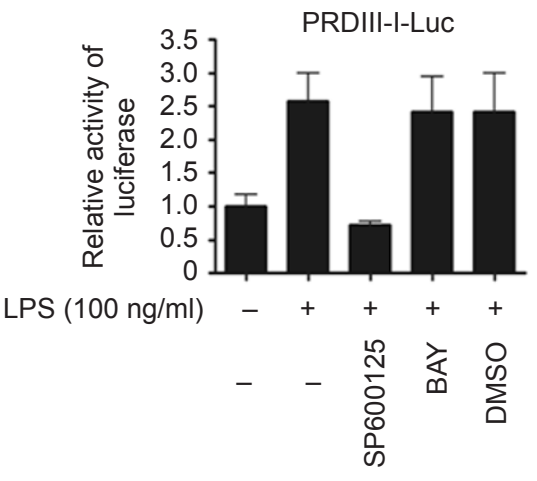

C

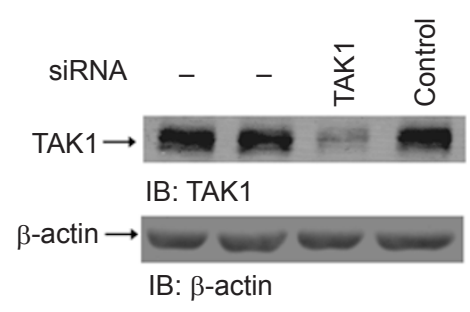

B

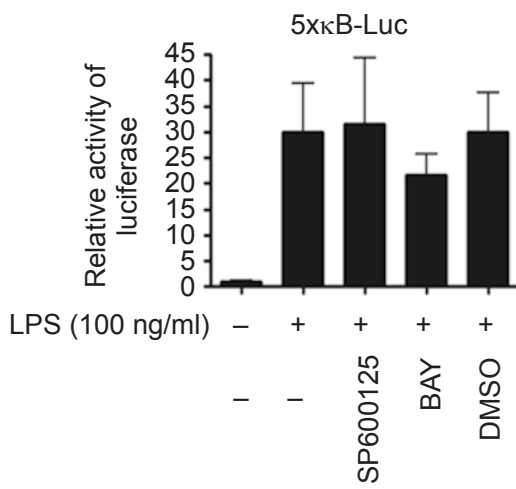

D

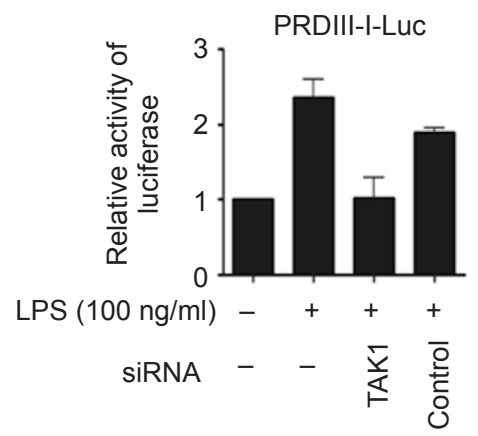

Figure 4 LPS-stimulated IRF3-responsive reporter gene activity is severely impaired by SP600125. Raw264.7 cells were transfected with reporter gene PRD III-I-Luc (A) or $5 \times_{\mathrm{K} B}$-Luc (B), along with pRL-SV40. The transfected cells were treated with the indicated inhibitors $1 \mathrm{~h}$ before and during LPS treatment for another $8 \mathrm{~h}$. Luciferase activities were measured as described under Materials and Methods. (C) TAK1 or control siRNA oligo was transfected into Raw264.7 cells. $48 \mathrm{~h}$ after transfection, cells were lysed and immunoblotted with anti-TAK1 (upper panels) or anti- $\beta$-actin (lower panels) antibody. (D) TAK1 or control siRNA oligo was co-transfected into Raw264.7 cells with PRD III-I-Luc and pRL-SV40. $48 \mathrm{~h}$ after transfection, cells were treated with LPS for $8 \mathrm{~h}$ and reporter assays were performed. 
influence IRF3-mediated endogenous gene expression induced by LPS and polyI:C. As shown in Figure 5A, inhibition of JNK activation by SP600125 completely abolished the expression of IFN- $\beta$ upon LPS stimulation. Because IFN- $\beta$ was regulated collectively by AP- 1 , NF$\kappa \mathrm{B}$ and IRF3 [40], this inhibition was due to the inactivation of both IRF3 and AP-1. To clearly demonstrate the function of IRF3 phosphorylation by JNK, we chose other two genes (RANTES and ISG15), which had been shown to be regulated primarily by IFN-stimulated response element (IRF3 target DNA sequence), but not by AP-1 [41, 42]. Blocking of JNK activation markedly attenuated the expression of RANTES and ISG15 induced by LPS. In contrast, BAY and DMSO had no inhibitory effect on their expression (Figure 5A). In addition, PD98059 and SB202190 had no inhibitory effect on the expression of IFN- $\beta$, RANTES, and ISG15 stimulated by LPS (data not shown). Alternatively, endogenous TAK1 was knocked down and the same inhibition was observed for the expression of these genes (IFN- $\beta$, RANTES, ISG15) (Figure 5C). Added to this, we carried out quantitative real-time PCR and confirmed that JNK and TAK1 contributed to the expression of IRF3 target genes (Figure $5 \mathrm{~B}$ and $5 \mathrm{D}$ ). Consistently, this was also the case for ISG15 expression stimulated by polyI:C (Figure 5E and $5 F)$. In contrast, IFN- $\beta$ could trigger ISG15 via the IRF3independent pathway [43], but this induction of ISG15 was not attenuated by inhibiting JNK activity (Figure $5 \mathrm{G})$. Taken together, these data indicate that the TAK1JNK cascade plays an important role in regulating IRF3 activation.

JNK regulates IRF3 activity by synergizing with the action of TBK1 kinase

Innate immune response can trigger the induction of IFN expression, in which phosphorylation of IRF3 by TBK1 is an essential regulatory process. Recent groundbreaking work has identified several key regulatory proteins that are critical for this process. For example, overexpression of TRIF, RIG-I, and MAVS has been shown to activate TBK1 and lead to IRF3 phosphorylation at its C-terminus [44]. The current study identified JNK to phosphorylate IRF3 at its N-terminal. Therefore, we wondered whether this phosphorylation could synergize with phosphorylation by TBK1. We found that inhibition of JNK activity markedly attenuated PRD III-I-driven gene expression induced by transfected (Figure 6A) or external(Figure 6B) polyI:C. In a similar manner, TRIF-, RIG-I-, and MAVS-mediated PRD III-I reporter gene activity was also reduced by SP600125 (Figure 6C-6E). Furthermore, IRF3 activation stimulated by TBK1 per se was consistently attenuated when JNK activity was im- paired (Figure 6F). Although the overexpression of these proteins could also activate $\mathrm{NF}-\kappa \mathrm{B}$, this inhibition did not apply to $\kappa \mathrm{B}$-driven gene expression induced by the same proteins (data not shown). These data suggest that TBK1 and MAPK signaling converged at IRF3; JNK and TBK1 cooperated with each other to achieve the maximum IRF3 activation.

Alternatively, we made use of IRF3(5D), a constitutively active form of IRF3, to bypass the function of TBK1 toward IRF3. The IRF3(5D) had previously been shown to induce IRF3-responsive reporter gene activation when overexpressed in cell lines [45]. Intriguingly, we found that IRF3(5D)-triggered PRD III-I-Luc activation was repressed specifically by SP600125, which indicated that JNK activity was indeed required for proper activation of IRF3 (Figure 7A). To directly demonstrate the synergy between TBK1 and JNK, we transfected PRD III-I-Luc into 293T cells along with different combinations of JNK1, JNK1 (APF), TAK1, TAK1 (KD), and IRF3(5D). As shown in Figure 7B, JNK1 or TAK1, but not JNK1 (APF) or TAK1 (KD), promoted the transcriptional activity of IRF3(5D) when co-expressed. More interestingly, JNK1 and polyI:C can synergize the activity of IRF3(5D) and S175A-IRF3(5D), but not that of S173A-IRF3(5D) (Figure 7C and 7D). In summary, these data supported the idea that robust activation of IRF3 depended on its phosphorylation at both the N- and C-terminal segments, which were mediated by JNK and TBK1, respectively.

Phosphorylation of IRF3 by JNK is essential for its dimerization induced by polyI: $C$

The above analysis suggested that JNK played a distinct role from TBK1 in phosphorylating IRF3. Our previous study had reported that forms III and IV of IRF3 phosphorylation induced by TBK1 were blocked by GA [46]. Interestingly, inhibition of JNK did not affect the appearance of forms III and IV of IRF3 (Figure 8A), which indicated that JNK and TBK1 phosphorylated IRF3 individually. Furthermore, polyI:C alone could induce both the 1-201 construct (data not shown) and endogenous IRF3 (Figure 8B) to change from form I to II, which indicated that $\mathrm{N}$-terminal phosphorylation of IRF3 took place in vivo during virus infection. This change was obviously mediated by JNK, because it was significantly attenuated by JNK inhibitor but not by BAY or DMSO. On the other hand, polyI:C failed to stimulate the generation of form II S173A-IRF3, also indicating that form II of IRF3 was mediated by JNK (Figure 8C). We then went on to explore what physiological process of IRF3 was regulated by JNK-mediated phosphorylation. In our experiments, polyI:C could induce IRF3 di- 
A

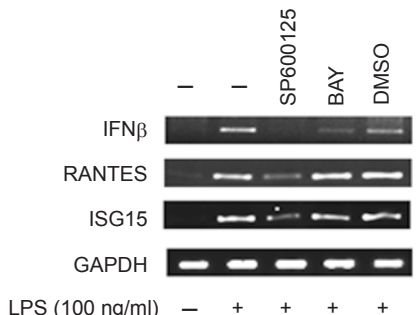

B
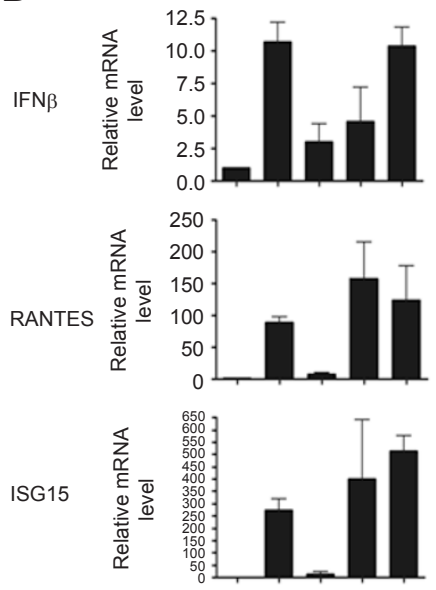

$\operatorname{LPS}(100 \mathrm{ng} / \mathrm{ml})++++$

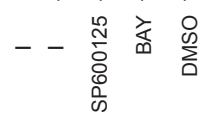

E

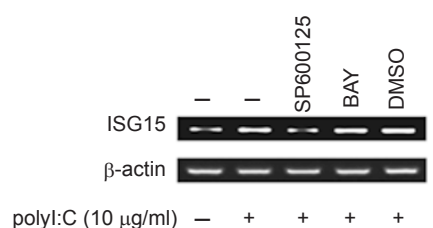

F

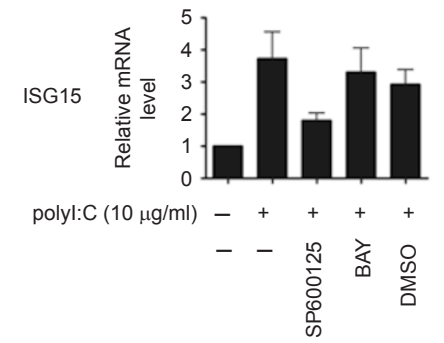

C

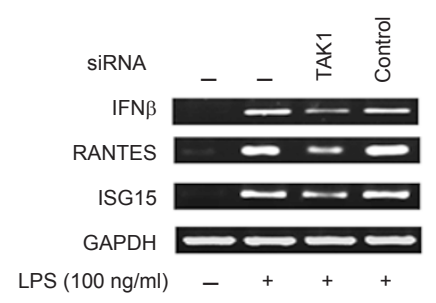

$\mathrm{FN} \beta$

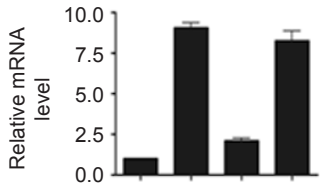

RANTES

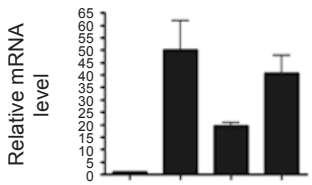

ISG15

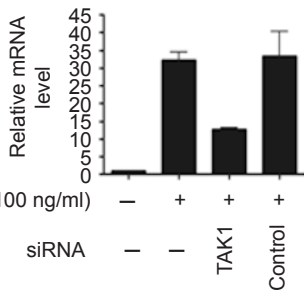

G

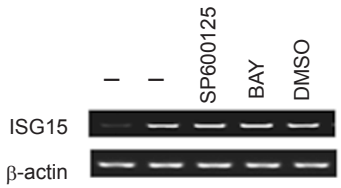

$\operatorname{IFN} \beta(100 \mu \mathrm{g} / \mathrm{ml})-++++$

Figure 5 IRF3-mediated gene expressions are severely impaired by SP600125 on LPS or polyl:C treatment. Raw264.7 (A, B), 293-TLR3 (E, F), or 293 (G) cells were pretreated with the indicated inhibitors for $1 \mathrm{~h}$ prior to challenge with LPS (A, B), polyl:C (E, F), or IFN $\beta(\mathbf{G})$ for eight more hours. Total RNAs were extracted and an equal amount of RNA was amplified by $\operatorname{RT}-\operatorname{PCR}(\mathbf{A}, \mathbf{E}, \mathbf{G})$ or quantitative real-time PCR (B, F) using specific primers for IFN $\beta$, RANTES, and ISG15, respectively. (C, D) TAK1 or control siRNA oligo was transfected into Raw264.7 cells. 48 h after transfection, cells were left treated with LPS for 8 h and RT-PCR assay (C) or quantitative real-time PCR (D) was performed as described above. Data represent means \pm SD (error bars) of at least three independent experiments. 
A

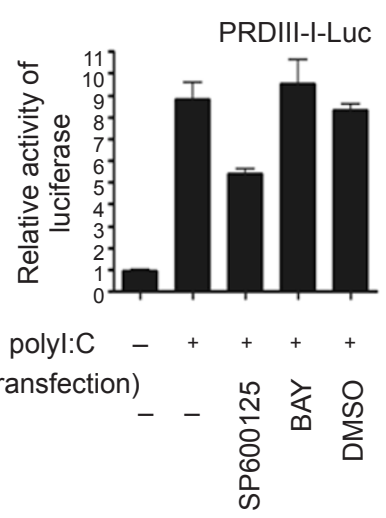

C

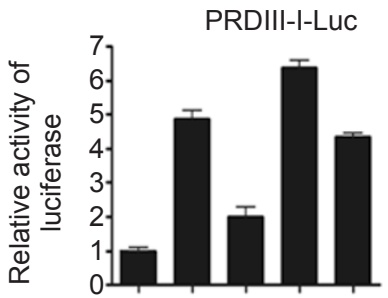

Flag-TRIF- + + + +

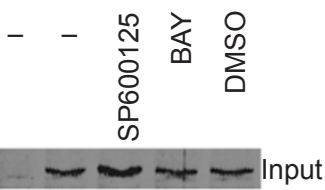

IB: Flag

E

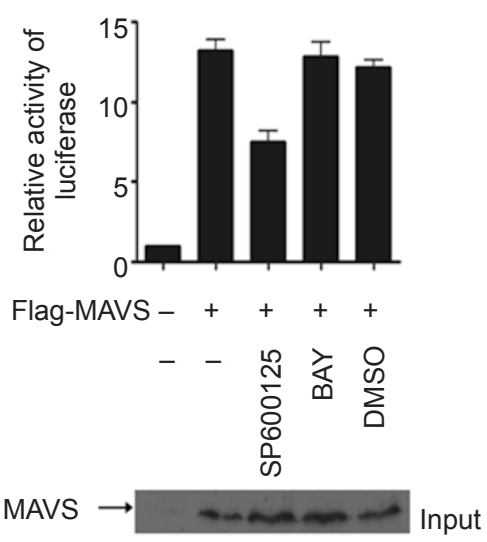

IB: Flag
B

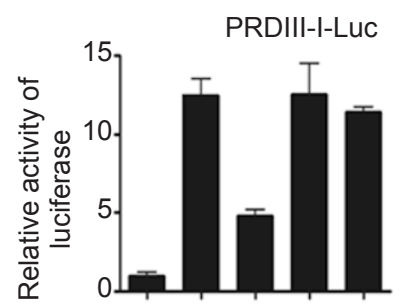

polyl: C -++++

(Medium)

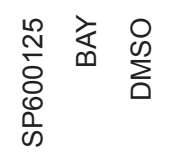

D

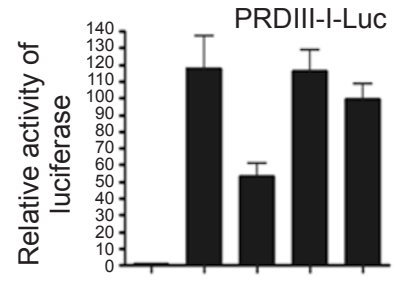

HA-RIG-I(N)- + + + +

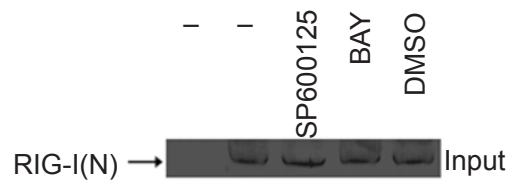

IB: HA

F

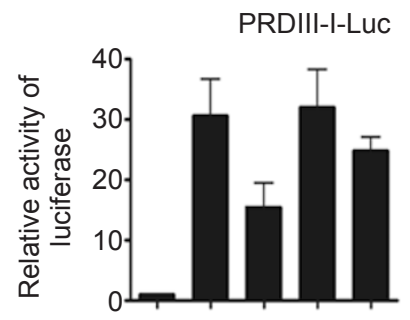

Flag-TBK1 ++++
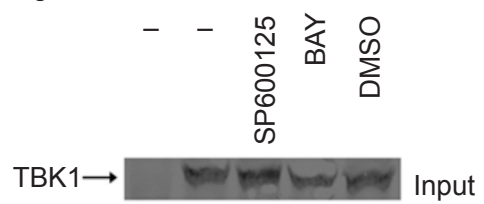

IB: Flag

Figure 6 The effect of SP600125 on IRF3 signaling. (A) $16 \mathrm{~h}$ after transfection with PRD III-I-Luc and pRL-SV40, polyl:C was transfected into 293T cells. After $12 \mathrm{~h}$, cells were treated with the indicated inhibitors for $8 \mathrm{~h}$ and luciferase activity was determined with the dual-luciferase assay system. (B) $16 \mathrm{~h}$ after transfection with PRDIII-I-Luc and pRL-SV40, polyl:C and various inhibitors were added into the medium of 293-TLR3 cells. After $12 \mathrm{~h}$, cells were harvested and luciferase activity was determined with the dual-luciferase assay system. TRIF (C), RIG-I(N) (D), MAVS (E), and TBK1 (F) were co-transfected into 293T cells with pRL-SV40 and reporter gene PRD III-I-Luc. $16 \mathrm{~h}$ after transfection, cells were treated with the indicated inhibitors for $8 \mathrm{~h}$ and luciferase activity was determined with the dual-luciferase assay system. 
A

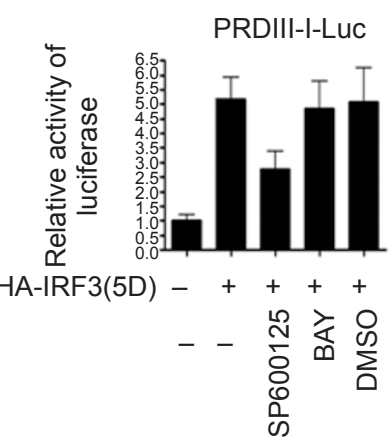

$\operatorname{IRF3(5D)\rightarrow } \underset{\text { IB: HA }}{\longrightarrow}$ Input
B

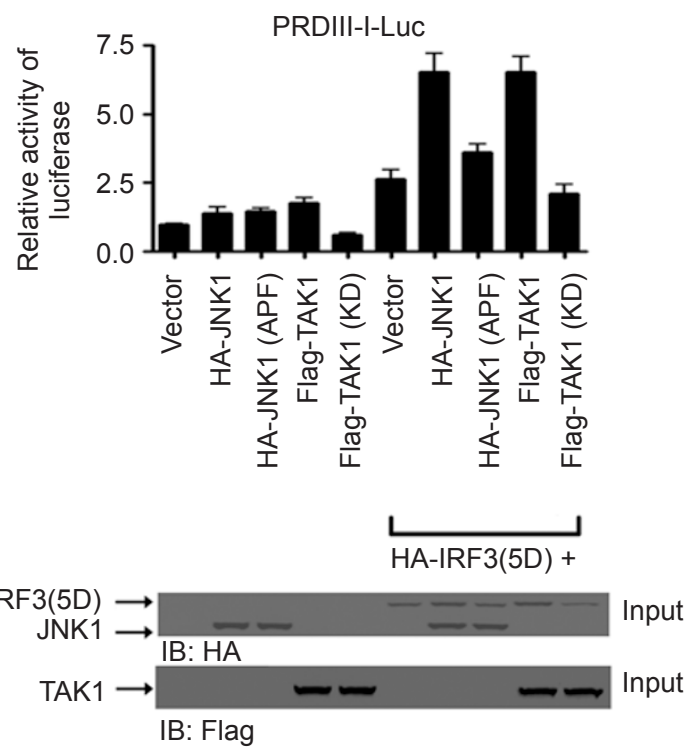

C
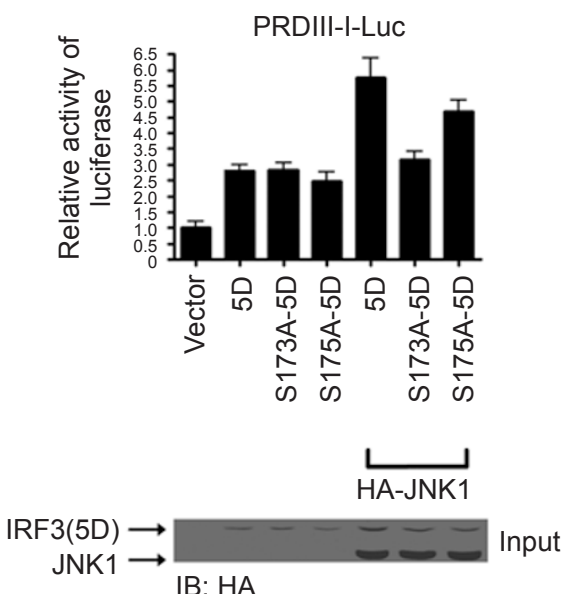

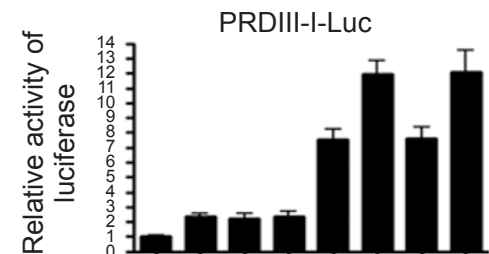

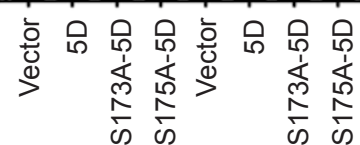

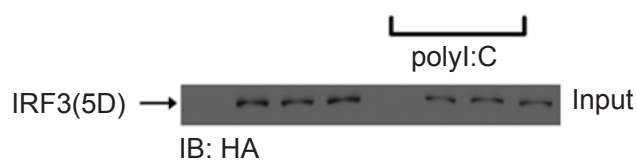

Figure 7 JNK can synergize the activation of IRF3(5D). (A) IRF3(5D) was co-transfected into 293T cells with pRL-SV40 and reporter gene PRDIII-I-Luc. $16 \mathrm{~h}$ after transfection, cells were treated with the indicated inhibitors for $8 \mathrm{~h}$ and luciferase activity was determined with the dual-luciferase assay system. (B) PRD III-I-Luc and pRL-SV40 were co-transfected into 293T cells along with various constructs, as indicated. $24 \mathrm{~h}$ after transfection, luciferase activities were measured. (C) PRD III-I-Luc and pRL-SV40 were co-transfected into 293T cells along with various constructs, as indicated. $24 \mathrm{~h}$ after transfection, luciferase activities were measured. Lower panels, the expression of various constructs. (D) PRD III-I-Luc and pRL-SV40 were cotransfected into 293-TLR3 cells along with various constructs, as indicated. $16 \mathrm{~h}$ after transfection, cells were treated with polyl:C as indicated for $8 \mathrm{~h}$ and then luciferase activities were measured.

merization, and this dimer disappeared completely when JNK activity was inhibited. This was not due to enhanced degradation, because cell lysates retained comparably the same amount of IRF3 in different treatments (Figure $8 \mathrm{D})$. Taken together, these data suggest that JNK could regulate the dimerization of IRF3 during virus infection and plays a complementary role in IRF3 activation with TBK1.

\section{Discussion}

The battle between the host and microbes is an ev- 
A

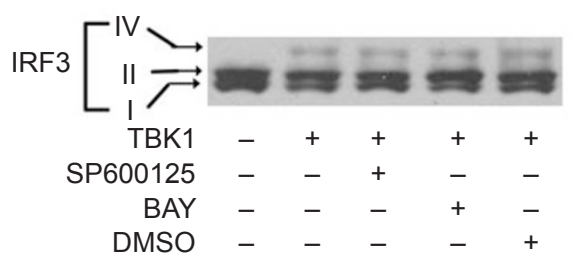

IB: IRF3
B

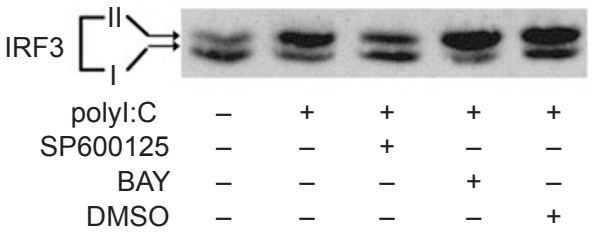

IB: IRF3

C

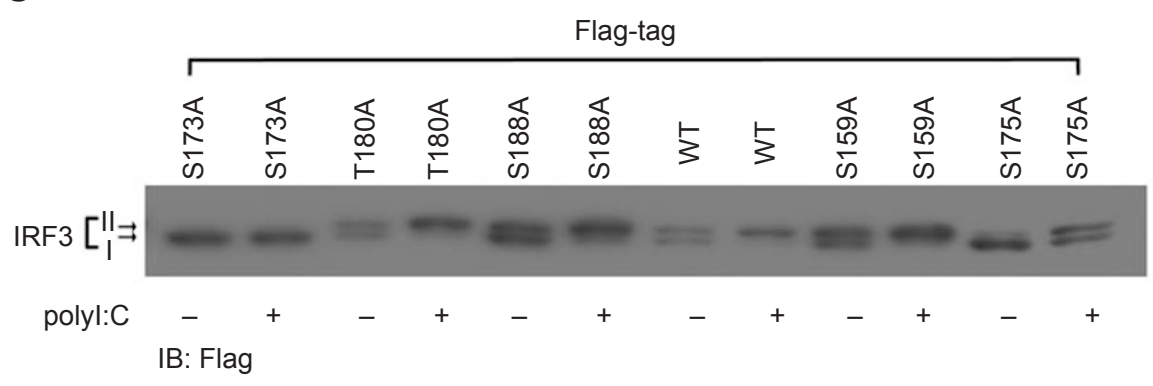

D

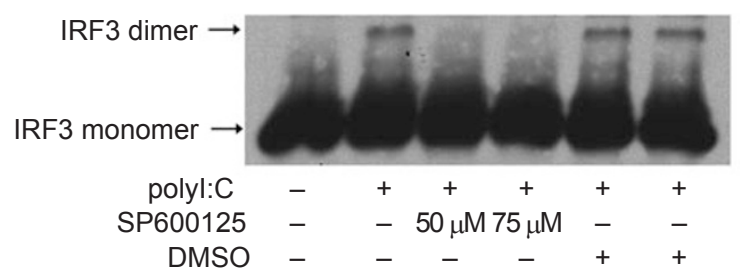

IB: IRF3

Figure 8 SP600125 dramatically abrogates IRF3 phosphorylation and dimerization stimulated by polyl:C. (A) $16 \mathrm{~h}$ after transfection of 293T cells with TBK1, cells were incubated with the indicated inhibitors for $8 \mathrm{~h}$ and harvested. Whole cell extracts were analyzed using anti-IRF3 antibody. (B) 293-TLR3 cells were treated with the indicated inhibitors $1 \mathrm{~h}$ before and during polyl:C treatment for an additional $1 \mathrm{~h}$. Whole cell extracts were resolved by $7.5 \%$ SDS-PAGE and immunoblotted using anti-IRF3 antibody. (C) 293-TLR3 cells were transiently transfected with various point mutants of IRF3, as indicated. $20 \mathrm{~h}$ after transfection, cells were stimulated with polyl:C as indicated for $1 \mathrm{~h}$ and then harvested. The cell lysates were immunoblotted using anti-Flag antibody. (D) 293-TLR3 cells were treated with SP600125 in the indicated concentrations $1 \mathrm{~h}$ before and during polyl: $C$ treatment for an additional $1 \mathrm{~h}$. Whole cell extracts were resolved by $7.5 \%$ native-PAGE and immunoblotted by using anti-IRF3 antibody.

erlasting theme in biomedical research. Breakthroughs have been made in recent years regarding how host cells survey the presence of microbes and trigger several signaling pathways (e.g., NF-kB, IRF3, MAPK) to initiate relevant innate and adaptive immune responses. Two receptor systems were demonstrated to play essential roles in the innate immune response, where these defense systems are physiologically and functionally interconnected. One is the TLR-dependent system, which is localized on the membrane of innate immune cells and monitors the presence of topologically extracellular mi- crobes. Another is the RLR and NLR-dependent system, which is localized inside the cytoplasm of host cells and monitors the presence of topologically intracellular microbes. For example, viral dsRNA could be recognized by TLR3, whereas bacterial lipopolysaccharides (LPS) could be recognized by TLR4. In addition, intracellular viruses and bacteria could be sensed by RIG-I/MDA5 and NOD1/NOD2, respectively [6, 44, 47]. Interestingly, one of the immediate consequences for some of these recognitions was the phosphorylation, dimerization, and translocation of transcription factor (IRF3), which were 
fundamental for the induction of type I IFNs. TBK1/ IKK $\varepsilon$ had been characterized as the critical converging kinases downstream of these receptor systems, which directly phosphorylated IRF3 via a cluster of serine /threonine residues at its $\mathrm{C}$-terminus. This phosphorylation could cause IRF3 translocation into the nucleus and its interaction with $\mathrm{CBP} / \mathrm{p} 300$, resulting in gene expression $[13,14]$.

In addition to TBK1/IKK, IRF3 was actively modulated via other processes. Several recent studies have revealed a novel mechanism for the termination of IRF3 activity. For example, IRF3 was targeted for ubiquitinproteasomal-mediated degradation following virus infection. Cullin1, a component of the ubiquitin ligase-stem cell factor complex, was required for formation of the polyubiquitin chain. This process was promoted by peptidyl-prolyl isomerase Pin1 via an unknown mechanism $[48,49]$. Interestingly, rotavirus-encoded protein NSP1 could induce IRF3 degradation and herpes simplex virus ICP0 could also inhibit IRF3 action [50, 51]. In contrast, proteolysis of IRF3 was counteracted by the induction of ISG15 expression, which could covalently modify IRF3 and provide a feedback mechanism [52]. In addition, IRF3 was recently shown to be modified by glutathione in resting cells and GRX-1 catalyzed the deglutathionylation step on virus infection [53]. However, the physiological significance of this post-translational modification remains to be explored.

As a primary transcriptional factor, IRF3 displayed four forms of phosphorylation status, among which forms III and IV were catalyzed by TBK1/IKK $\beta$, whereas form I was un-phosphorylated. However, the kinase(s) responsible for the generation of form II and whether any physiological significance exists for this phosphorylation remain unknown. In this study, we characterized JNK to be the kinase for form II IRF3 and this phosphorylation was important for IRF3 dimerization. This conclusion was supported by several lines of evidence, as reiterated below. First, the expression of JNK could convert IRF3 from form I to II, and this was enhanced by the upstream kinase TAK1. ERK1, ERK2, p38, and other kinases could not achieve this effect. Second, recombinant or endogenous JNK (data not shown) stimulated by LPS or polyI:C could phosphorylate IRF3 per se. This phosphorylation took place at the N-terminus of IRF3, which was distinct from phosphorylation of the $\mathrm{C}$-terminus mediated by TBK1. Third, the JNK-specific inhibitor SP600125 could abolish greatly the generation of form II IRF3 without affecting the action of TBK1 on IRF3, whereas other kinase inhibitors could not. Similarly, the point mutation of serine 173 to alanine blocked the phosphorylation of IRF3 by JNK. Fourth, anti-microbial gene expressions (e.g., IFN- $\beta$, RANTES, ISG15) were severely attenuated when JNK activity was downregulated. This observation was further confirmed by reporter gene expression that was responsive only to IRF3. Fifth, knockdown of TAK1 obtained significant attenuation of IRF3 activation in terms of its target genes and generation of form II phosphorylation. Unfortunately, we failed to knock down both JNK1 and JNK2 simultaneously and were unable to directly explore the effects of the loss of function of JNKs. Sixth, the signaling pathway leading to TBK1 was parallel to that leading to JNK. Both the kinases synergized with each other to achieve maximal IRF3 activation. This was consistent with the result indicating that TBK1 and JNK1 targeted different domains of IRF3. How this synergy is fulfilled with respect to IRF3 structure still remains to be determined. Seventh, polyI:C alone could induce endogenous IRF3 to become form II. This took place at the $\mathrm{N}$-terminal serine 173 residue and could be inhibited by JNK inhibitor. Importantly, polyI:C could induce dimerization of IRF3, which was impaired by SP600125. Thus, this investigation established a new layer of regulation of IRF3 activation by the TAK1-JNK cascade.

Our current finding was in line with an earlier report that DNA damage could cause N-terminal phosphorylation of IRF3. Interestingly, this report had mapped a phosphorylation region similar to ours. However, the authors did not reveal which kinase was responsible for the phosphorylation. In particular, the former study did not explore whether the phosphorylation had any physiological relevance on DNA damage [17]. In addition, Kim et $a l$. reported that DNA damage per se could activate IRF3 and induce corresponding defensive genes. However, they did not investigate the role of N-terminal phosphorylation. Instead, they observed that S385/386 at the Cterminus of IRF3 was important for IRF3 activation during DNA damage [54]. As DNA damage could robustly activate JNK, it is likely that JNK is responsible for IRF3 N-terminal phosphorylation during DNA damage. This would suggest a unified model for cellular stress response and highlight the important role of JNK in IRF3 activation. In addition, it would be intriguing to explore whether TBK1/IKK $\varepsilon$ is activated on DNA damage, what the common signaling molecules upstream of JNK for both DNA damage and microbial invasion might be, and what the shared response elicited by IRF3 is.

Interestingly, a growing list of kinases was implicated to have a role in IRF3 activation. For example, MEKK1, PI3K-AKT, cPKCs and ASK1 kinase had been reported to participate in the modulation of IRF3 activity [26-28, 40]. However, they did not directly target IRF3 per se and their functions were most likely indirect and secondary, 
resulting from cross-talk between signaling pathways of stress responses. A similar case occurred in NF- $\kappa \mathrm{B}$ activation, which was shown to be modulated by approximately a dozen important kinases [55]. Addressing how the actions of these kinases are inter-related and elucidating the molecular basis of their synergy or inhibition are challenging.

In summary, this investigation revealed that the TAK1-JNK cascade was required for IRF3 function in addition to $\mathrm{TBK} 1 / \mathrm{IKK} \varepsilon$, which uncovered a new mechanism for the MAPK in regulation of innate immunity. We thus propose a tentative model: when microbes attack host cells, their PAMPs (pathogen-associated molecular patterns) are immediately detected by host PRRs (PAMP responsive receptors), which initiate several signaling pathways that converge on IRF3 as well as other transcription factors. In particular, JNK and TBK1 directly phosphorylate IRF3 at its N- and C-terminal domains, respectively, which then dimerizes, translocates into the nucleus, and interacts with $\mathrm{CBP} / \mathrm{p} 300$ to induce robust gene expressions. The cytokines and chemokines produced can restrict the spread of microbial agents to other cells and recruit immune cells to eliminate the microbes.

\section{Materials and Methods}

\section{Reagents}

PolyI:C (GE Healthcare) was diluted in endotoxin-free sterile $\mathrm{H}_{2} \mathrm{O}$ (Invitrogen) and used at $10 \mu \mathrm{g} / \mathrm{ml}$. LPS was purchased from Sigma-Aldrich and used at $100 \mathrm{ng} / \mathrm{ml}$. Hu-IFN- $\beta$ (mammalian) was obtained from PBL Biomedical Laboratories and used at 100 $\mu \mathrm{g} / \mathrm{ml}$. The JNK inhibitor SP600125 (Sigma-Aldrich) and NF- $\mathrm{KB}$ inhibitor BAY 11-7082 (KangChen Bio-tech) were used at $50 \mu \mathrm{M}$ and $10 \mu \mathrm{M}$, respectively. The specific ERK inhibitor PD98059 and p38 inhibitor SB202190 were purchased from Calbiochem (Merck) and used at $50 \mu \mathrm{M}$. Other commercially available reagents and antibodies used were as follows: protein $\mathrm{A} / \mathrm{G}$ beads, HA, IRF3, TAK1, ERK and phospho-ERK antibodies were purchased from Santa Cruz Biotechnology, Inc. Flag and $\beta$-actin antibodies were obtained from Sigma-Aldrich. Phospho-p38 antibody was purchased from BD Biosciences. JNK antibody was purchased from R\&D Systems. TAK1 siRNA duplexes were chemically synthesized by GenePharma. The TAK1 siRNA sequence was GUGCUG ACA UGU CUG AAA UdTdT; the control siRNA sequence was UUC UCC GAA CGU GUC ACG UdTdT. GST-IRF3 and GST-IRF3(S173A) were purified from Escherichia coli by glutathione Sepharose ${ }^{\mathrm{TM}}$ 4B (Pharmacia Biotech).

\section{Plasmids}

pcDNA3.1-N-Flag was a gift from Dr Hai Wu from the UT Southwestern Medical Center and contained a Flag-tag at the Nterminal sequence. pcDNA3.1-N-Flag-TBK1, pcDNA3.1-N-FlagIRF3, and truncation mutants of IRF3 were described previously [46]. The point mutants of IRF3 (S159A; S173A; S175A; T180A; S188A) and IRF3(5D) (S173A-IRF3(5D) and S175A-IRF3(5D)) were constructed using a Quikchange XL site-directed mutagene- sis method. The pcDNA3-N-HA-IRF3 was obtained by subcloning IRF3 cDNA into the pcDNA3-N-HA vector. The cDNA encoding constitutively active IRF3(5D) was a gift from Professor John Hiscott (McGill University, Montreal, Quebec, Canada) and was subcloned into the pcDNA3.1-N-Flag vector and pcDNA3-N-HA vector. pcDNA3-Flag-p38, pCEP4-ERK1, and pCEP4-ERK2 were kindly provided by Professor Melanie Cobb from the University of Texas Southwest Medical Center. Plasmids of human JNK1, JNK2, and JNK1 (APF) were kindly provided by Professor Lin Li (Shanghai Institute of Biochemistry and Cell Biology, Shanghai, China). cDNAs encoding TAK1, TAK1 (KD), TAB1, IKK $\beta$, and the reporter genes $(5 \times \kappa \mathrm{B}$-Luc and pRL-SV40) have been described previously [56]. The (PRDIII-I)4-Luc reporter gene construct was kindly provided by Professor Stephan Ludwig (Heinrich-Heine-University, Duesseldorf, Germany). In addition, FlagMAVS was a gift from Professor Hong-Bing Shu (College of Life Sciences, Wuhan University, Wuhan, China). Human TRIF and RIG-I (N) cDNAs were obtained by PCR from the human thymus plasmid cDNA library (Clontech). The pcDNA3.1-N-Flag-TRIF and pcDNA3-N-HA-RIG-I (N) were constructed by subcloning cDNAs into pcDNA3.1-N-Flag or pcDNA3-N-HA vector. All constructs were confirmed by automatic DNA sequencing.

\section{Cell culture and transfection}

Human embryonic kidney 293T cells, 293-TLR3 cells, and mouse RAW264.7 cells were cultured using DMEM (Invitrogen) supplemented with $10 \%$ FBS (Hyclone), penicillin $(50 \mathrm{U} / \mathrm{ml})$, and streptomycin $(50 \mu \mathrm{g} / \mathrm{ml})$. The $293-\mathrm{TLR} 3$ cells were provided by Zhengfan Jiang (Beijing University). All transfections were performed using LipofectAMINE 2000 (Invitrogen) according to the manufacturer's instructions. Cells were harvested at different times after transfection, as indicated.

\section{Reporter assays}

Cells were seeded in 12-well plates and transfected with the indicated plasmids. The total amount of DNA was kept constant by supplementing with pcDNA3. pRL-SV40 (Promega) was included in all transfection experiments as an internal control to correct for transfection efficiency. At $16 \mathrm{~h}$ after transfection, cells were pretreated with inhibitors for $1 \mathrm{~h}$ prior to challenge with the indicated reagents and harvested after eight additional hours of incubation. Luciferase activity was analyzed with the Dual Luciferase Reporter Assay System (Promega) using Berthold Detection Systems (Sirius, Germany). Data represent means \pm SD (error bars) of at least three independent experiments.

\section{Real-time RT-PCR}

Total RNA was isolated with TRIzol (Invitrogen) as per the manufacturer's instructions. Reverse transcription of purified RNA was performed using oligonucleotide $\mathrm{dT}$ primer. The quantification of gene transcripts was performed by real-time PCR using SYBR green I dye (Invitrogen). Expression values were normalized with control GAPDH or $\beta$-actin. The primers used were as follows: mus IFN- $\beta$, sense (CTT CTC CAC CAC AGC CCT CTC) and antisense (CCC ACG TCA ATC TTT CCT CTT); mus RANTES, sense (ATG AAG ATC TCT GCA GCT GCC) and antisense (TAGGCA AAG CAG CAG GGA GTG); mus ISG15, sense (CACAGT GAT GCT AGT GGT AC) and antisense (CTT AAG CGTGTC TAC AGT CTG); mus GAPDH, sense (GAA GGG CTCATG ACC ACA GT) and antisense (GGA TGC AGG GAT GAT- 
GTT CT); human ISG15, sense (TGT CGG TGT CAG AGCTGA AG) and antisense (GCC CTT GTT ATT CCT CAC CA); human $\beta$-actin, sense (AAA GAC CTG TAC GCC AAC AC) and antisense (GTC ATA CTC CTG CTT GCT GAT). Product size was verified by agarose gel electrophoresis, and melt curve analysis was performed to check primer specificity. Data represent means \pm SD (error bars) of at least three independent experiments.

\section{Immunoblotting}

Cells were rinsed twice in PBS, harvested, pelleted by centrifugation and solubilized at $4{ }^{\circ} \mathrm{C}$ in lysis buffer $(1 \%$ Nonidet P-40, $20 \mathrm{mM}$ Tris, pH 7.5, $20 \mathrm{mM} \beta$-glycerol phosphate, $10 \mathrm{mM} \mathrm{NaF}$, $0.5 \mathrm{mM} \mathrm{Na}_{3} \mathrm{VO}_{4}, 150 \mathrm{mM} \mathrm{NaCl}, 1 \mathrm{mM}$ DTT, $1 \mathrm{mM}$ PMSF, and $0.2 \mathrm{mM}$ EDTA, $\mathrm{pH} 7.0$, plus a protease inhibitor cocktail). After $15 \mathrm{~min}$, lysates were clarified by centrifugation at $4{ }^{\circ} \mathrm{C}, 15000 \times g$, for $15 \mathrm{~min}$. The supernatant was resolved on SDS-PAGE gels and transferred to polyvinylidene difluoride membranes (Millipore), blocked with $5 \%$ milk in TBS- $0.05 \%$ Tween-20 for $1 \mathrm{~h}$ and incubated overnight with various primary antibodies. Membranes were washed with TBS-Tween-20 and incubated for $1 \mathrm{~h}$ with APconjugated (Promega) or horseradish peroxidase-linked (Jackson ImmunoResearch Laboratories, Inc.) secondary antibodies, and the bound antibodies were visualized using the NBT/BCIP Western Blotting System (Promega) or Supersignal West Pico Chemiluminescence ECL Kit (Pierce).

\section{In vitro kinase assay}

Cell pellets (Raw 264.7 or 293 T cell expressed with HA-tagged JNK1/2) were lysed and harvested. For immunoprecipitation, cell lysates were first mixed with anti-HA antibody in the respective tubes for $2 \mathrm{~h}$ at $4{ }^{\circ} \mathrm{C}$, followed by the addition of $20 \mu \mathrm{l}$ of protein A/G beads (Santa Cruz Technology) for another $1 \mathrm{~h}$ at $4{ }^{\circ} \mathrm{C}$. Beads were washed twice in lysis buffer and twice in JNK reaction buffer (25 mM Hepes, $\mathrm{pH} 7.4,10 \mathrm{mM} \mathrm{MgCl}, 1 \mathrm{mM}$ dithiothreitol, $0.1 \mathrm{mM}$ EDTA). Each sample was added to $20 \mu \mathrm{l}$ of in vitro JNK assay buffer containing $5 \mu \mathrm{Ci}$ of $\left[{ }^{32} \mathrm{P}\right] \mathrm{ATP}, 50 \mu \mathrm{M}$ of cold ATP, and $2 \mu \mathrm{g}$ of purified GST-IRF3 (WT) or GST-IRF3 (S173A). Mixed samples were incubated for $30 \mathrm{~min}$ at $30{ }^{\circ} \mathrm{C}$. Reactions were terminated with the addition of $6 \times$ SDS loading buffer, and proteins were subjected to SDS-PAGE. Phosphorylation of IRF3 was visualized using a PhosphorImager (Molecular Dynamics).

\section{Native PAGE assay}

Cells were harvested as described before and a $2 \times$ native sample buffer (100 mM Tris-Cl, pH 6.8, 30\% glycerol, and 2\% deoxycholate was added to the lysates, which contained an equal amount of total proteins $(20 \mu \mathrm{g})$. $7.5 \%$ gels (without SDS) were pre-run with $25 \mathrm{mM}$ Tris and $192 \mathrm{mM}$ glycine, $\mathrm{pH} 8.3$, with and without $1 \%$ deoxycholate in the cathode and anode chamber, respectively, for $1 \mathrm{~h}$ at $10 \mathrm{~mA}$ and $4{ }^{\circ} \mathrm{C}$. Subsequently, samples were applied to the gel and electrophoresed for $\sim 1 \mathrm{~h}$ at $20 \mathrm{~mA}$ and $4{ }^{\circ} \mathrm{C}$ and transferred to polyvinylidene difluoride membranes (Millipore) for $1 \mathrm{~h}$ at $270 \mathrm{~mA}$ and $4{ }^{\circ} \mathrm{C}$.

\section{Acknowledgments}

We thank Prof. John Hiscott (McGill University, Canada), Melanie Cobb (University of Texas Southwest Medical Center, USA), Stephan Ludwig (Heinrich-Heine-University, Duessel- dorf, Germany) and Hong-Bing Shu (College of Life Sciences, Wuhan University, Wuhan, China) for providing plasmids for this study. We thank Prof. Zhengfan Jiang (Beijing University, China) for providing the 293-TLR3 cell line. This study is partly supported by grants from the Chinese Academy Renovation Program (KSCX1-YW-R-06), the Ministry of Science and Technology of China (2002CB513000, 2006AA02Z121 and 2007CB914504) and the National Natural Science Foundation of China (30225013, 30623003 and 30521005).

\section{References}

1 Akira S, Takeda K, Kaisho T. Toll-like receptors: critical proteins linking innate and acquired immunity. Nat Immunol 2001; 2:675-680.

2 Takeda K, Akira S. Toll-like receptors in innate immunity. Int Immunol 2005; 17:1-14.

3 Akashi S, Shimazu R, Ogata H, et al. Cutting edge: cell surface expression and lipopolysaccharide signaling via the toll-like receptor 4-MD-2 complex on mouse peritoneal macrophages. J Immunol 2000; 164:3471-3475.

4 Alexopoulou L, Holt AC, Medzhitov R, Flavell RA. Recognition of double-stranded RNA and activation of NF-kappaB by Toll-like receptor 3. Nature 2001; 413:732-738.

5 Oshiumi H, Matsumoto M, Funami K, Akazawa T, Seya T. TICAM-1, an adaptor molecule that participates in Toll-like receptor 3-mediated interferon-beta induction. Nat Immunol 2003; 4:161-167.

6 Meylan E, Tschopp J, Karin M. Intracellular pattern recognition receptors in the host response. Nature 2006; 442:39-44.

7 O'Neill LA. Toll-like receptor signal transduction and the tailoring of innate immunity: a role for Mal? Trends Immunol 2002; 23:296-300.

8 Sato S, Sugiyama M, Yamamoto M, et al. Toll/IL-1 receptor domain-containing adaptor inducing IFN-beta (TRIF) associates with TNF receptor-associated factor 6 and TANK-binding kinase 1, and activates two distinct transcription factors, NFkappa B and IFN-regulatory factor-3, in the Toll-like receptor signaling. J Immunol 2003; 171:4304-4310.

9 Takeuchi O, Akira S. Toll-like receptors; their physiological role and signal transduction system. Int Immunopharmacol 2001; 1:625-635.

10 Yamamoto M, Sato S, Hemmi H, et al. Role of adaptor TRIF in the MyD88-independent toll-like receptor signaling pathway. Science 2003; 301:640-643.

11 Oshiumi H, Sasai M, Shida K, Fujita T, Matsumoto M, Seya T. TIR-containing adapter molecule (TICAM)-2, a bridging adapter recruiting to toll-like receptor 4 TICAM-1 that induces interferon-beta. J Biol Chem 2003; 278:49751-49762.

12 Yamamoto M, Sato S, Hemmi H, et al. TRAM is specifically involved in the Toll-like receptor 4-mediated MyD88-independent signaling pathway. Nat Immunol 2003; 4:1144-1150.

13 Fitzgerald KA, McWhirter SM, Faia KL, et al. IKKepsilon and TBK1 are essential components of the IRF3 signaling pathway. Nat Immunol 2003; 4:491-496.

14 Sharma S, tenOever BR, Grandvaux N, Zhou GP, Lin R, Hiscott J. Triggering the interferon antiviral response through an IKK-related pathway. Science 2003; 300:1148-1151.

15 Hemmi H, Takeuchi O, Sato S, et al. The roles of two Ikap- 
paB kinase-related kinases in lipopolysaccharide and double stranded RNA signaling and viral infection. J Exp Med 2004; 199:1641-1650.

16 McWhirter SM, Fitzgerald KA, Rosains J, Rowe DC, Golenbock DT, Maniatis T. IFN-regulatory factor 3-dependent gene expression is defective in Tbk1-deficient mouse embryonic fibroblasts. Proc Natl Acad Sci USA 2004; 101:233-238.

17 Servant MJ, ten Oever B, LePage C, et al. Identification of distinct signaling pathways leading to the phosphorylation of interferon regulatory factor 3. J Biol Chem 2001; 276:355-363.

18 Yoneyama M, Suhara W, Fujita T. Control of IRF-3 activation by phosphorylation. J Interferon Cytokine Res 2002; 22:73-76.

19 Suhara W, Yoneyama M, Iwamura T, et al. Analyses of virusinduced homomeric and heteromeric protein associations between IRF-3 and coactivator CBP/p300. J Biochem 2000; 128:301-307.

20 Yoneyama M, Suhara W, Fukuhara Y, Fukuda M, Nishida E, Fujita T. Direct triggering of the type I interferon system by virus infection: activation of a transcription factor complex containing IRF-3 and CBP/p300. EMBO J 1998; 17:1087-1095.

21 Collins SE, Noyce RS, Mossman KL. Innate cellular response to virus particle entry requires IRF3 but not virus replication. $J$ Virol 2004; 78:1706-1717.

22 Clement JF, Bibeau-Poirier A, Gravel SP, et al. Phosphorylation of IRF-3 on Ser 339 generates a hyperactive form of IRF3 through regulation of dimerization and CBP association. $J$ Virol 2008; 82:3984-3996.

23 Dauber B, Schneider J, Wolff T. Double-stranded RNA binding of influenza B virus nonstructural NS1 protein inhibits protein kinase $\mathrm{R}$ but is not essential to antagonize production of alpha/ beta interferon. J Virol 2006; 80:11667-11677.

24 Spiegel M, Pichlmair A, Martinez-Sobrido L, et al. Inhibition of beta interferon induction by severe acute respiratory syndrome coronavirus suggests a two-step model for activation of interferon regulatory factor 3. J Virol 2005; 79:2079-2086.

25 Han KJ, Su X, Xu LG, Bin LH, Zhang J, Shu HB. Mechanisms of the TRIF-induced interferon-stimulated response element and NF-kappaB activation and apoptosis pathways. $J$ Biol Chem 2004; 279:15652-15661.

26 Sarkar SN, Peters KL, Elco CP, Sakamoto S, Pal S, Sen GC. Novel roles of TLR3 tyrosine phosphorylation and PI3 kinase in double-stranded RNA signaling. Nat Struct Mol Biol 2004; 11:1060-1067.

27 Johnson J, Albarani V, Nguyen M, Goldman M, Willems F, Aksoy E. Protein kinase Calpha is involved in interferon regulatory factor 3 activation and type I interferon-beta synthesis. $J$ Biol Chem 2007; 282:15022-15032.

28 Chiang E, Dang O, Anderson K, Matsuzawa A, Ichijo H, David $M$. Cutting edge: apoptosis-regulating signal kinase 1 is required for reactive oxygen species-mediated activation of IFN regulatory factor 3 by lipopolysaccharide. J Immunol 2006; 176:5720-5724.

29 Jiang Z, Mak TW, Sen G, Li X. Toll-like receptor 3-mediated activation of NF-kappaB and IRF3 diverges at Toll-IL-1 receptor domain-containing adapter inducing IFN-beta. Proc Natl Acad Sci USA 2004; 101:3533-3538.

30 Lewis TS, Shapiro PS, Ahn NG. Signal transduction through MAP kinase cascades. Adv Cancer Res 1998; 74:49-139.

31 Liu MK, Herrera-Velit P, Brownsey RW, Reiner NE. CD14- dependent activation of protein kinase $\mathrm{C}$ and mitogen-activated protein kinases (p42 and p44) in human monocytes treated with bacterial lipopolysaccharide. J Immunol 1994; 153:26422652.

32 Rawadi G, Ramez V, Lemercier B, Roman-Roman S. Activation of mitogen-activated protein kinase pathways by $\mathrm{Myco}$ plasma fermentans membrane lipoproteins in murine macrophages: involvement in cytokine synthesis. J Immunol 1998; 160:1330-1339.

33 Ono K, Ohtomo T, Sato S, et al. An evolutionarily conserved motif in the TAB1 C-terminal region is necessary for interaction with and activation of TAK1 MAPKKK. J Biol Chem 2001; 276:24396-24400.

34 Shibuya H, Yamaguchi K, Shirakabe K, et al. TAB1: an activator of the TAK1 MAPKKK in TGF-beta signal transduction. Science 1996; 272:1179-1182.

35 Bennett BL, Sasaki DT, Murray BW, et al. SP600125, an anthrapyrazolone inhibitor of Jun N-terminal kinase. Proc Natl Acad Sci USA 2001; 98:13681-13686.

36 Koedel U, Bayerlein I, Paul R, Sporer B, Pfister HW. Pharmacologic interference with NF-kappaB activation attenuates central nervous system complications in experimental Pneumococcal meningitis. J Infect Dis 2000; 182:1437-1445.

37 Cao Z, Xiong J, Takeuchi M, Kurama T, Goeddel DV. TRAF6 is a signal transducer for interleukin-1. Nature 1996; 383:443446.

38 Shirakabe K, Yamaguchi K, Shibuya H, et al. TAK1 mediates the ceramide signaling to stress-activated protein kinase/c-Jun N-terminal kinase. J Biol Chem 1997; 272:8141-8144.

39 Ehrhardt C, Kardinal C, Wurzer WJ, et al. Rac1 and PAK1 are upstream of IKK-epsilon and TBK-1 in the viral activation of interferon regulatory factor-3. FEBS Lett 2004; 567:230-238.

40 Kim T, Kim TY, Lee WG, Yim J, Kim TK. Signaling pathways to the assembly of an interferon-beta enhanceosome. Chemical genetic studies with a small molecule. J Biol Chem 2000; 275:16910-16917.

41 Lin R, Heylbroeck C, Genin P, Pitha PM, Hiscott J. Essential role of interferon regulatory factor 3 in direct activation of RANTES chemokine transcription. Mol Cell Biol 1999; 19:959-966.

42 Au WC, Moore PA, Lowther W, Juang YT, Pitha PM. Identification of a member of the interferon regulatory factor family that binds to the interferon-stimulated response element and activates expression of interferon-induced genes. Proc Natl Acad Sci USA 1995; 92:11657-11661.

43 van Boxel-Dezaire AH, Rani MR, Stark GR. Complex modulation of cell type-specific signaling in response to type I interferons. Immunity 2006; 25:361-372.

44 Seth RB, Sun L, Chen ZJ. Antiviral innate immunity pathways. Cell Res 2006; 16:141-147.

45 Heylbroeck C, Balachandran S, Servant MJ, et al. The IRF-3 transcription factor mediates Sendai virus-induced apoptosis. $J$ Virol 2000; 74:3781-3792.

46 Yang K, Shi H, Qi R, et al. Hsp90 regulates activation of interferon regulatory factor 3 and TBK-1 stabilization in Sendai virus-infected cells. Mol Biol Cell 2006; 17:1461-1471.

47 Meurs EF, Breiman A. The interferon inducing pathways and the hepatitis C virus. World J Gastroenterol 2007; 13:24462454. 
48 Bibeau-Poirier A, Gravel SP, Clement JF, et al. Involvement of the IkappaB kinase (IKK)-related kinases tank-binding kinase $1 / \mathrm{IKKi}$ and cullin-based ubiquitin ligases in IFN regulatory factor-3 degradation. J Immunol 2006; 177:5059-5067.

49 Saitoh T, Tun-Kyi A, Ryo A, et al. Negative regulation of interferon-regulatory factor 3-dependent innate antiviral response by the prolyl isomerase Pin1. Nat Immunol 2006; 7:598-605.

50 Graff JW, Ewen J, Ettayebi K, Hardy ME. Zinc-binding domain of rotavirus NSP1 is required for proteasome-dependent degradation of IRF3 and autoregulatory NSP1 stability. J Gen Virol 2007; 88:613-620.

51 Lin R, Noyce RS, Collins SE, Everett RD, Mossman KL. The herpes simplex virus ICP0 RING finger domain inhibits IRF3and IRF7-mediated activation of interferon-stimulated genes. $J$ Virol 2004; 78:1675-1684.
$52 \mathrm{Lu} \mathrm{G}$, Reinert JT, Pitha-Rowe I, et al. ISG15 enhances the innate antiviral response by inhibition of IRF-3 degradation. Cell Mol Biol (Noisy-le-grand) 2006; 52:29-41.

53 Prinarakis E, Chantzoura E, Thanos D, Spyrou G. S-glutathionylation of IRF3 regulates IRF3-CBP interaction and activation of the IFNbeta pathway. EMBO J 2008; 27:865-875.

54 Kim T, Kim TY, Song YH, Min IM, Yim J, Kim TK. Activation of interferon regulatory factor 3 in response to DNAdamaging agents. J Biol Chem 1999; 274:30686-30689.

55 Viatour P, Merville MP, Bours V, Chariot A. Phosphorylation of NF-kappaB and IkappaB proteins: implications in cancer and inflammation. Trends Biochem Sci 2005; 30:43-52.

56 Diao L, Zhang B, Xuan C, et al. Activation of c-Jun N-terminal kinase (JNK) pathway by HSV-1 immediate early protein ICP0. Exp Cell Res 2005; 308:196-210. 